International Journal of Pure and Applied Mathematics

Volume 88 No. 4 2013, 547-555

ISSN: 1311-8080 (printed version); ISSN: 1314-3395 (on-line version)

url: http://www.ijpam.eu

doi: http://dx.doi.org/10.12732/ijpam.v88i4.9

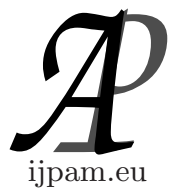

\title{
EXPLICIT BOND OPTION IN HEATH JARROW MORTON MODEL WITH CONSTANT VOLATILITY
}

\author{
Abbes Benchaabane ${ }^{1 \S}$, Azzedine Benchettah ${ }^{2}$ \\ ${ }^{1}$ Laboratoire of Applied Mathematics and Modeling \\ University of Guelma \\ BP 401, Guelma, 24000, ALGERIA \\ ${ }^{2}$ Laboratoire of Numerical Analysis, \\ Optimization and Statistics \\ University of Annaba \\ BP 12, Annaba, 23000, ALGERIA
}

\begin{abstract}
The purpose of this paper is to investigate the Heath Jarrow Morton model (HJM) with constant volatility to provide an explicit formula for European option on zero-coupon bonds.
\end{abstract}

AMS Subject Classification: 91G30, 91B26, $91 \mathrm{~B} 70$

Key Words: interest rates, market models, stochastic models

\section{Introduction}

There has been an accelerated growth in the use of interest rate instruments in fixed income portfolios and risk management systems over the past two decades. With this proliferation has emerged the necessity to formulate reliable models that accurately price and hedge such interest rate sensitive cash flows.

The literature on interest rate modelling is dominated by two main groups of models; the short rate and forward rate model. The Vasicek model [8] and the Cox-Ingersoll-Ross model [1] relies in the fact that prices are explicit functions of the instantaneous 'spot' interest rate so that these models are unable to take

Received: September 9, 2013

(C) 2013 Academic Publications, Ltd.

${ }^{\S}$ Correspondence author url: www.acadpubl.eu 
the whole yield curve observed on the market into account in the price structure [6]. Some authors have resorted to a two-dimensional analysis to improve the models in terms of discrepancies between short and long rates, e.g. Scaefer and Schwartz [7]. These more complex models do not lead to explicit formulae and require the solution of partial differential equations [5].

More recently, Ho and Lee [3] have proposed a discrete time model describing the behaviour of the whole yield curve. The continuous time model we present is based on the same idea and has been introduced by Heath, Jarrow and Morton [2]. These models are extremely popular due to their simplicity and mathematical tractability. The HJM one factor model essentially assumes that the term structure is affected by a single source of uncertainly.

The aim of this study is to present a Heath, Jarrow, and Morton model where the Volatility is a constant. Furthermore, it is to give an explicit formula for European option on zero-coupon bonds.

\section{Generalities}

We take as given a Brownian motion $\left\{W(t) ; 0 \leq t \leq T^{*}\right\}$ an some probability space $(\Omega, \mathcal{F}, P)$, and $\left\{F(t) ; 0 \leq t \leq T^{*}\right\}$ is the filtration generated by $W$. The market we will study is mainly the market of zero coupon bonds

Definition 1. A zero coupon bond with maturity date $T$, also called a $T$-bond, is contract which guarantees the holder $1 \$$ to be paid on the date $T$. The price at time $t$ of a bond with maturity date $T$ is denoted by $B(t, T)$. Obviously we have $B(T, T)=1$.

We now make an assumption to guarantee the existence of a sufficiently rich bond market

Definition 2. 1. The forward rate for $[S, T]$ contracted at $t$ is defined as

$$
R(t ; S, T)=-\frac{\log B(t, T)-\log B(t, S)}{T-S} .
$$

2. The spot rate, $R(S, T)$, for the period $[S, T]$ is defined as

$$
R(S, T)=R(S ; S, T)
$$

3. The instantaneous forward rate with maturity $T$, contracted at $t$ is defined by

$$
f(t, T)=-\frac{\partial \log B(t, T)}{\partial T} .
$$


4. The instantaneous short rate at time $t$ is defined by

$$
r(t)=f(t, t)
$$

As an immediate consequence of the definitions we have the following useful formulas

Lemma 3. For $t \leq s \leq T$ we have

$$
B(t, T)=\exp \left\{-\int_{t}^{T} f(t, s) d s\right\} .
$$

We note this as an alternative representation for $B(t, T)$ by contrast with its expression in terms of the short rate process $r$ :

$$
B(t, T)=E\left[\exp \left(-\int_{t}^{T} r(s) d s\right) \mid \mathcal{F}_{t}\right]
$$

\section{The Heath-Jarrow-Morton Model}

The Heath-Jarrow-Morton (HJM) model for term structure considers stochastic differential equations for the evolution of the forward rate $f(t, T)$.

For each $\left.T \in] 0, T^{*}\right]$ suppose the dynamics of $f$ are given by

$$
d f(t, T)=\alpha(t, T) d t+\sigma(t, T) d W(t)
$$

Here the coefficients $\alpha(u, T)$ and $\sigma(u, T)$, for $0 \leq u \leq T$, are measurable (in $(u, w))$ and adapted. The integral form of $(7)$ is

$$
f(t, T)=f(0, T)+\int_{0}^{t} \alpha(u, T) d u+\int_{0}^{t} \sigma(u, T) d W(u) .
$$

Note we have two time parameters and recall

$$
B(t, T)=\exp \left\{-\int_{t}^{T} f(t, u) d u\right\} .
$$

With $d$ denoting a differential in the $t$ variable:

$$
d\left\{-\int_{t}^{T} f(t, u) d u\right\}=f(t, t) d t-\int_{t}^{T}(d f(t, u)) d u
$$




$$
\begin{aligned}
& =r(t) d t-\int_{t}^{T}[\alpha(t, u) d t+\sigma(t, u) d W(t)] d u \\
& =\left(r(t)-\alpha^{*}(t, T)\right) d t+\sigma^{*}(t, T) d W(t),
\end{aligned}
$$

where

$$
\begin{aligned}
\alpha^{*}(t, T) & =\int_{t}^{T} \alpha(t, u) d u \\
\sigma^{*}(t, T) & =\int_{t}^{T} \sigma(t, u) d u .
\end{aligned}
$$

Recall, by definition, $f(t, u)$ is an $\mathcal{F}_{t}$-adapted process. Therefore,

$$
X(t):=-\int_{t}^{T} f(t, u) d u
$$

is an $\mathcal{F}_{t}$-adapted process. In fact it is an It process with,

$$
d X(t)=\left(r(t)-\alpha^{*}(t, T)\right) d t-\sigma^{*}(t, T) d W(t) .
$$

Also, $B(t, T)=e^{X(t)}$, so

$$
\begin{aligned}
d B(t, T) & =e^{X(t)}\left[r(t)-\alpha^{*}(t, T)+\frac{1}{2} \sigma^{*}(t, T)^{2}\right] d t-e^{X(t)} \sigma^{*}(t, T) d W(t) \\
& =B(t, T)\left[\left(r(t)-\alpha^{*}(t, T)+\frac{1}{2} \sigma^{*}(t, T)^{2}\right) d t-\sigma^{*}(t, T) d W(t)\right] .
\end{aligned}
$$

Now, the discounted $B(t, T)$ will be a martingale under $P$ (so $P$ is a risk-neutral measure), if for $0 \leq t \leq T \leq T^{*}$,

$$
\alpha^{*}(t, T)=\frac{1}{2}\left(\sigma^{*}(t, T)\right)^{2} .
$$

from the definitions of $\alpha^{*}$ and $\sigma^{*}$ this means

$$
\int_{t}^{T} \alpha(t, u) d u=\frac{1}{2}\left(\int_{t}^{T} \sigma(t, u) d u\right)^{2} .
$$

This is equivalent to

$$
\alpha(t, T)=\sigma(t, T) \int_{t}^{T} \sigma(t, u) d u .
$$

If $P$ itself is not a risk-neutral measure there may be a probability $P^{\lambda}$ under which $\left(\exp \int_{0}^{t}-r(u) d u\right) B(t, T)$ is a martingale. This is the content of the following result due to Heath, Jarrow and Morton [2]. 
Theorem 4. For each $\left.T \in] 0, T^{*}\right]$ suppose $\alpha(u, T)$ and $\sigma(u, T)$ are adapted processes. We assume $\sigma(u, T)>0$ for all $u, T$, and $f(0, T)$ is a deterministic function of $T$. The instantaneous forward rate $f(t, T)$ is defined by

$$
f(t, T)=f(0, T)+\int_{0}^{t} \alpha(u, T) d u+\int_{0}^{t} \sigma(u, T) d W(u) .
$$

Then the term structure model determined by the processes $f(t, T)$ does not allow arbitrage if and only if there is an adapted process $\lambda(t)$ such that

$$
\alpha(t, T)=\sigma(t, T) \int_{t}^{T} \sigma(t, u) d u+\sigma(t, T) \lambda(t), \text { for all } 0 \leq t \leq T \leq T^{*},
$$

and the process

$$
\Lambda^{\lambda}(t):=\exp \left(-\int_{0}^{t} \lambda(u) d W(u)-\frac{1}{2} \int_{0}^{t} \lambda(u)^{2} d u\right)
$$

is an $\left(\mathcal{F}_{t}, P\right)$ martingale.

\section{Application of HJM Model when $\sigma$ is a Constant}

In the HJM model, we assume that the function $\sigma$ is a positive constant. We assume that there is an underlying probability space $(\Omega, \mathcal{F}, P)$, equipped with a standard filtration $\mathcal{F}_{t}$ Under the risk-neutral measure $P$, we would like to price a call with maturity $\theta$ and strike price $K$, on a zero-coupon bond with maturity $T>\theta$.

Under the risk-neutral measure $P$, the instantaneous forward rate dynamics is given by

$$
f(t, u)=f(0, u)+\sigma^{2} t(u-t / 2)+\sigma W_{t} .
$$

The price of zero-coupon bond with maturity $T$ at time $\theta$ is

$$
\begin{aligned}
B(\theta, T) & =\exp \left\{-\int_{\theta}^{T} f(\theta, u) d u\right\} \\
& =\frac{\exp \left\{-\int_{0}^{T} f(\theta, u) d u\right\}}{\exp \left\{-\int_{0}^{\theta} f(\theta, u) d u\right\}} \\
& =\frac{\exp \left\{-\int_{0}^{T} f(0, u) d u\right\} \exp \left\{-\int_{0}^{T}\left[\sigma^{2} \theta(u-\theta / 2)+\sigma W_{\theta}\right] d u\right\}}{\exp \left\{-\int_{0}^{\theta} f(0, u) d u\right\} \exp \left\{-\int_{0}^{\theta}\left[\sigma^{2} \theta(u-\theta / 2)+\sigma W_{\theta}\right] d u\right\}}
\end{aligned}
$$




$$
=\frac{B(0, T)}{B(0, \theta)} \exp \left(-\sigma(T-\theta) W_{\theta}-\frac{\sigma^{2} \theta T(T-\theta)}{2}\right) .
$$

We have, for $\lambda \in \mathbf{R}$

$$
\begin{aligned}
& E\left[\exp \left\{-\sigma \int_{0}^{\theta} W_{s} d s\right\} \exp \left\{\lambda W_{\theta}\right\}\right]=E\left[\exp \left\{-\sigma \int_{0}^{\theta} W_{s} d s+\lambda W_{\theta}\right\}\right] \\
& =\exp \left\{\frac{1}{2}\left[\begin{array}{ll}
-\sigma & \lambda
\end{array}\right]\left[\begin{array}{cc}
\frac{\theta^{3}}{3} & \frac{\theta^{2}}{2} \\
\frac{\theta^{2}}{2} & \theta
\end{array}\right]\right\} \\
& =\exp \left\{\frac{1}{2}\left(\frac{\sigma^{2} \theta^{3}}{3}-\lambda \sigma \theta^{2}+\lambda^{2} \theta\right)\right\} \text {. }
\end{aligned}
$$

We define a new probability measures $P_{1}$ and $P_{2}$ with densities with respect to the risk-neutral measure $P$ respectively by setting

$$
\begin{aligned}
\frac{d P_{1}}{d P} & =\frac{\exp \left\{-\int_{0}^{\theta} r(s) d s\right\} B(\theta, T)}{B(0, T)}, \\
\frac{d P_{2}}{d P} & =\frac{\exp \left\{-\int_{0}^{\theta} r(s) d s\right\} B(\theta, T)}{B(0, \theta)} .
\end{aligned}
$$

Using the above result we have, $\forall \lambda \in$ textbf $R$

$$
\begin{aligned}
E^{P_{1}}\left[\exp \left\{\lambda W_{\theta}\right\}\right]= & E\left(\frac{e^{-\int_{0}^{\theta} r(s) d s} B(\theta, T)}{B(0, T)} \exp \left\{\lambda W_{\theta}\right\}\right) \\
= & \exp \left(\frac{-\sigma^{2} \theta T(T-\theta)}{2}-\frac{1}{6} \sigma^{2} \theta^{3}\right) \times \\
& \times \exp \left(\frac{1}{6} \sigma^{2} \theta^{3}-\frac{1}{2}\left(\lambda-\sigma(T-\theta) \sigma \theta^{2}+\frac{1}{2}(\lambda-\sigma(T-\theta))^{2}\right) \theta\right. \\
= & \exp \left[\lambda\left(\sigma \theta(T-\theta)-\frac{\sigma \theta^{2}}{2}\right)+\frac{1}{2} \lambda^{2} \theta\right] .
\end{aligned}
$$

It follows that, under the probability $P_{1}, W_{\theta}$ is a normal random with mean

$$
E^{P_{1}}\left(W_{\theta}\right)=\sigma \theta(T-\theta)-\frac{\sigma \theta^{2}}{2}
$$

and variance

$$
\operatorname{Var}^{P_{1}}\left(W_{\theta}\right)=\theta
$$


Similarly, $\forall \lambda \in \mathbf{R}$

$$
\begin{aligned}
E^{P_{2}}\left[\exp \left\{\lambda W_{\theta}\right\}\right] & =E\left(\frac{e^{-\int_{0}^{\theta} r(s) d s}}{B(0, \theta)} \exp \left\{\lambda W_{\theta}\right\}\right) \\
& =\exp \left[\lambda\left(-\frac{\sigma \theta^{2}}{2}\right)+\frac{1}{2} \lambda^{2} \theta\right] .
\end{aligned}
$$

Then, under the probability $P_{2}, W_{\theta}$ is a normal random with mean

$$
E^{P_{2}}\left(W_{\theta}\right)=-\frac{\sigma \theta^{2}}{2}
$$

and variance

$$
\operatorname{Var}^{P_{2}}\left(W_{\theta}\right)=\theta
$$

We know that, the price of call with maturity $\theta$ and strike price $K$ at time 0 , on a zero-coupon bond with maturity $T>\theta$ is define as

$$
C_{0}=E\left[e^{-\int_{0}^{\theta} r(s) d s}(B(\theta, T)-K)^{+}\right] .
$$

We have

$$
\begin{aligned}
B(\theta, T)-K & \geq 0 \\
& \Longrightarrow \frac{B(0, T)}{B(0, \theta)} \exp \left(-\sigma(T-\theta) W_{\theta}-\frac{\sigma^{2} \theta T(T-\theta)}{2}\right)-K \geq 0 \\
& \Longrightarrow W_{\theta} \leq d_{1},
\end{aligned}
$$

with

$$
d_{1}=-\frac{\sigma \theta T}{2}-\frac{\log \left(K \frac{B(0, \theta)}{B(0, T)}\right)}{\sigma(T-\theta)} .
$$

Consequently,

$$
\begin{aligned}
C_{0}= & \int_{\Omega} \chi_{\left\{x \leq d_{1}\right\}} \exp \left\{-\int_{0}^{\theta} r(s) d s\right\}(B(\theta, T) d P \\
& -\int_{\Omega} \chi_{\left\{x \leq d_{1}\right\}} \exp \left\{-\int_{0}^{\theta} r(s) d s\right\} K d P \\
= & \int_{\Omega} \chi_{\left\{x \leq d_{1}\right\}} B(0, T) d P_{1}-\int_{\Omega} \chi_{\left\{x \leq d_{1}\right\}} B(0, \theta) d P_{2} \\
= & B(0, T) \int_{-\infty}^{d_{1}} \frac{1}{\sqrt{2 \pi \theta}} \exp \left\{-\frac{1}{2}\left(x-\sigma \theta(T-\theta)+\frac{\sigma \theta^{2}}{2}\right)^{2}\right\} d x
\end{aligned}
$$




$$
-K B(0, \theta) \int_{-\infty}^{d_{1}} \exp \left\{-\frac{1}{2 \theta}\left(x+\frac{\sigma \theta^{2}}{2}\right)\right\} d x
$$

Therefore,

$$
C_{0}=B(0, T) \mathcal{N}\left(\frac{d_{1}-\sigma \theta(T-\theta)+\frac{\sigma \theta^{2}}{2}}{\sqrt{\theta}}\right)-K B(0, \theta) \mathcal{N}\left(\frac{d_{1}+\sigma \theta^{2}}{\sqrt{\theta}}\right) .
$$

Let use set

$$
d=\frac{\sigma \sqrt{\theta}(T-\theta)}{2}-\frac{\log \left(K \frac{B(0, \theta)}{B(0, T)}\right)}{\sigma \sqrt{\theta}(T-\theta)} .
$$

Using these notation, we have

$$
C_{0}=B(0, T) \mathcal{N}(d)-K B(0, \theta) \mathcal{N}(d-\sigma \sqrt{\theta}(T-\theta))
$$

where

$$
\mathcal{N}(\zeta)=\frac{1}{\sqrt{2 \pi}} \int_{-\infty}^{\zeta} \exp \left(-\frac{x^{2}}{2}\right) d x
$$

\section{References}

[1] John Cox, Jonathan Ingersoll, and Stephen Ross. A theory of the term structure of interest rates. Econometrica, 53(2):385-407, March 1985.

[2] David Heath, Robert Jarrow, and Andrew Morton. Bond pricing and the term structure of interest rates: A new methodology for contingent claims valuation. Econometrica: Journal of the Econometric Society, pages 77$105,1992$.

[3] Thomas SY Ho and SANG-BIN LEE. Term structure movements and pricing interest rate contingent claims. The Journal of Finance, 41(5):10111029, 1986.

[4] John Hull and Alan White. Pricing interest-rate-derivative securities. Review of financial studies, 3(4):573-592, 1990.

[5] Ioannis Karatzas and Steven E Shreve. Methods of mathematical finance, volume 39. Springer, 1998.

[6] Damien Lamberton. Introduction to stochastic calculus applied to finance. CRC press, 2008. 
[7] Stephen M Schaefer and Eduardo S Schwartz. A two-factor model of the term structure: An approximate analytical solution. Journal of Financial and Quantitative analysis, 19(4):413-424, 1984.

[8] Oldrich Vasicek. An equilibrium characterization of the term structure. Journal of financial economics, 5(2):177-188, 1977. 
